\title{
Investigation of the Effect of Electromagnetic Radiation on Human Health Using Remote Sensing Technique
}

\author{
Mohammed Mejbel Salih \\ Laser and optoelectronics engineering department, University of Technology-Iraq, Baghdad 10066, Iraq
}

Corresponding Author Email: 40339@uotechnology.edu.iq

https://doi.org/10.18280/ijsse.110113

Received: 27 August 2020

Accepted: 3 February 2021

\section{Keywords:}

EMF radiations, remote sensing, $R F$ field

power meter, human health

\begin{abstract}
In the previous two decades, there has been a rapid and remarkable development in the field of communication technologies to encompass many joints of social life, especially devices for daily use, from mobile phones to laptops, to microwave transmitting and receiving towers, in addition to electromagnetic induction furnaces. This puts us in the fact that we are currently inside a multi-spectrum electromagnetic cloud. In this research, the effect of exposure to electromagnetic radiation and checking the negative side effects on the human body was studied through the use of remote sensing techniques, an electromagnetic radiation intensity measuring device for some devices circulating daily with humans, i.e. mobile phones, to assess the effect of this radiation emitted on human health. The study adopts elementary standards to determine the value of the radioactive energy and its effect on human organs after taking samples from cell phones. In addition, the results show that the effects of radiation depend on depends on the time of exposure.
\end{abstract}

\section{INTRODUCTION}

It has become clear to everyone that there is an acceleration and a race in the development of new technologies that work with excellent efficiency that facilitate the process of circulation and simple use, as there are many engineering devices and equipment that provide many services to the world, and we mention, for example, communication technology and information exchange in which the principle of work depends on electromagnetic mobility. On the other hand, cannot be directly conceal the damage caused by this energy to all organs of the human body. Therefore, it has become important to study the theoretical fundamentals of the electromagnetic radiation spectrum and the possible negative effects on human health.

As a one of the modern sciences, remote sensing techniques have many applications. Also, it is very important to mention here that it is depended on obtaining information without direct contact on the body and to obtaining accurate data. For example, it adopted the technology of the compute of the radiant power from mobile phones using a device that is part of the remote sensing techniques.

Numerous studies have been made through using Remote sensing technique in many applications that relating to environmental issues. Adeola et al. [1] employed Remote sensing technique to determine breeding habitats. The study employed Nkomazi municipality as a case study. In Makurdi Town of Benue State, Hemba et al. [2] have examined the physical growth and expansion Using remote sensing technique. In another respect, Adefisan et al. [3] used a remote sensing technique to set areas vulnerable to flood hazard. The study selected Ibadan Metropolis as a case study for achieving this goal. Ige et al. [4] examined the use of remote sensing to detect changes in land cover in Abuja and all factors affecting the thermal environment. In order to reduce the impact on ecological sustainability, Onamuti et al. [5] used remote sensing to estimate the level of the shrinkage of Chad Lake.

Genuis [6] discussed the relationship between environmental medicine, human exposure and adverse health outcomes in most health education programs. The study showed that there is a large lack of information in environmental medicine on the risks to users of modern technology. Using Sprague-Dawley rats, Jorge-Mora et al. [7] revealed that acute exposure to electromagnetic fields caused an imbalance in anatomical heat shock protein levels. The distinctive feature of this research was the need to increase the number of researches concerned with monitoring and detecting the effects of electromagnetic radiation, especially that affecting the human body and raising its temperature above the normal range, resulting in many problems and fatal diseases. Ghezel-Ahmadi et al. [8] investigated the hypothesis of a link between electrohypersensitive and heavy metal exposure and demonstrated that heavy metal loads could play a role in exceptional cases. The investigation and analysis of the relationship between residential and occupational exposure to electromagnetic field was done by Baldi et al. [9]. the said study uncovered that occupational or residential exposure to extremely low frequency may play a role in the occurrence of meningioma. Yakymenko et al. [10] indicated that radiofrequency exposure-induced oxidative stress should be considered one of the key biology mechanisms of radiation operation.

Kostoff et al. [11] reported that the radiofrequency radiation exposure limits set by regulatory agencies are not fully protective against serious adverse health effects. These results have been supported by Melnick [12] who showed that the radiofrequency radiation had nigitive effects on human health.

According to the above survey, a lot of studies employed the remote sensing technique in different application, but there is no study using this technique to study the radiant power of 
the mobile phones and their effects on human health. In this paper, the theoretical fundamentals of the electromagnetic radiation spectrum and the possible negative effects of electromagnetic wave use by defining electromagnetic waves with an indication of their physical properties and biological effects on humans have been studied, as well as to the pathological symptoms that may affect users of these devices. This study employed a remote sensing technique, i.e. a device for measuring the intensity of electromagnetic radiation represented by the power of the radiation emitted towards the human body, to know the radiant power of the mobile phones.

\section{THEORETICAL CONCEPTS}

\subsection{Electromagnetic radiations}

In the year 1820, the scientist Orsted noticed that if a current passed through a wire, it would create a lot of magnetic. Also, he observed the movement of the iron particles and how it was organized. This shows the effect of electromagnetic radiation on the material and that all radiations exist with different names in all bands of spectrum have an effect on a specific substance according to the frequency and wavelength. On the other hand, electromagnetic spectrum such as visible light, microwave and X-rays, gamma rays and television and radio waves are all rays known as electromagnetic radiation. They are all the same properties, but they differ in wavelength and frequency, as is evident in the figure. In order to better understand these interactions, we must learn about the physical properties of microwave waves within the general electromagnetic spectrum as shown in the Figure 1.

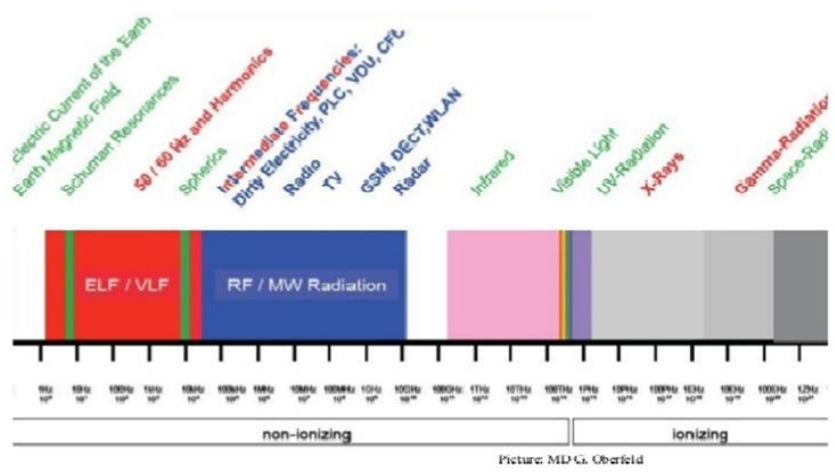

Figure 1. Electromagnetic bands

\subsection{Physical property and biological effect}

Electromagnetic fields are waves that travel at a speed of 300,000 kilometers per second, Figure 2, and carry energy. These fields consist of two fields spread in opposite directions of the same plane called the electric field and magnetic field. According to Figure 2, these three factors have relations with each other. Then, characteristic impedance, that is. Z, can be expressed as a constant ratio, which depends on the strength of the electric and magnetic fields, as shown in the equation below:

$$
Z=E / H
$$

Then, the magnitude and direction of the electromagnetic flux density can be represented by the energy vector as follows:

$$
S=E \times H
$$

The above equations can be simplified by substituting Eq. (1) into Eq. (2) where the value of $\mathrm{Z}$ is $120 \pi$ (i.e. 377 ):

$$
S=E^{2} / 120 \pi=E^{2} / 377
$$

and

$$
S=120 \pi H^{2}=377 H^{2}
$$

The frequency of the electromagnetic waves is defined as the number of cycles in one second that passes through a fixed point, the frequency has increased, as for microwave ovens, a frequency is used $2.45 \mathrm{GHz}$ and wavelength here equal $12 \mathrm{~cm}$.

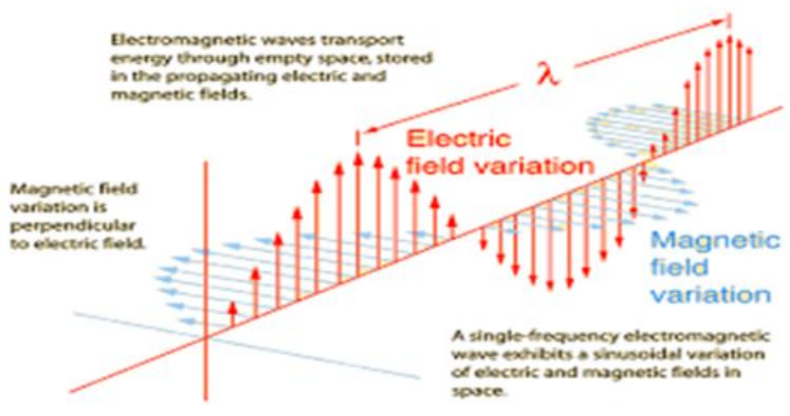

Figure 2. Electromagnetic variation

When we talk about what is caused by exposure to electromagnetic radiation, the effect of the first is a noticeable increase in thermal, that is, a noticeable increase in the temperature of the body's organs. In nature it responds to a certain frequency without others, for example that the frequency (2.4 5) $\mathrm{GHz}$ affects the fats and liquid materials in the cell, its high frequency range is of the non-follow-up type. If it falls on any substance, its fall on the cells contributes to the increase of negative charges, which in turn break down the capital, the link between the cells.

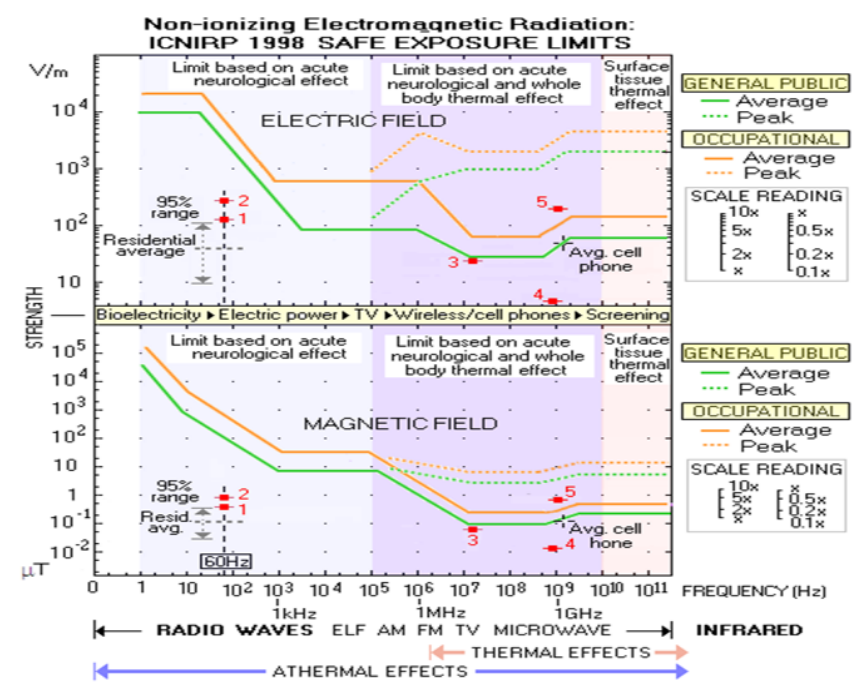

Figure 3. EMF radiations' effects

There is a relationship that links the value of the frequency 
with skin depth, and here we understand that the ability to create means to overcome the opposite energy, which causes agitation in the cells and increases their random movement, and this is caused by heat. Despite our talk about the manifestations of radiation exposure, there are many benefits to this exposure that contribute to improving many of the positives for humans produce vitamin $\mathrm{D}$ on the other hand. Some biological effects cause health damage such as pain resulting from sunburn or skin cancer, Figure 3.

\section{THE EFFECT OF ELECTROMAGNETIC RADIATION ON HUMAN HEALTH}

\subsection{Diseases brain and cancer}

Researcher David and his team discovered at the University of Nottingham after exposing some microorganisms continuously to short waves, including simple worms that effecting of biological composition easily. The larvae that have been exposed to a continuous dose for 12 hours for ultrasound have grown at a speed of more than five percent over those that have not been subjected to the same conditions, and this acceleration of growth may indicate the effect of short waves on the speed of cell division. Therefore, the research team is conducting the same experiment on a Breast animal see the effect of short waves on cell division. This raises concerns about the ability of these short waves to rapidly split cancer cells. In this way, concerns have reappeared about the impact of the use of mobile phones on the brain thanks to the facts revealed by J.S. and his team at the Research and Evaluation Laboratories at the US Department of Defense in Burton. This team exposed a section of the brain from very short waves in the experimental mouse and found that the electrical signals in the brain, it has changed somewhat and has poor response or ability to stimulate it. On the other hand, one of the most surprising facts came from the study currently known as amnesia, which was published in the International Journal of Bio-Radiations. This study installed a device that emits short waves similar to those issued by a mobile phone near the permission of people who volunteered to conduct experiments on them, and found that these people are capable to remember the words and pictures that were shown to them on the computer screens without any influence of the waves that emanate from the experimental devices. Accordingly, there are indications that were reached that the very short waves have made neurons in some experiments more capable and faster to respond to the variables related to the memory. In addition, the inventor of the mobile phone chips, the German chemist Friedelheim Wollenhorst, warned of the dangers of leaving mobile devices open in the bedrooms on the human brain and said that keeping these devices or any satellite transmitters or receiving devices in the bedroom causes a state of restlessness, anxiety, lack of sleep and brain damage which leads in the long run, it destroys the body's immune system.

He stressed that the radiation emitted from the mobile phone booster stations is equivalent in strength to the radiation from a small nuclear reactor and that the electromagnetic frequencies produced by the mobile phone are stronger than the X-rays that penetrate all parts of the body, and indicated that it can emit from the mobile energy higher than the permissible for the tissues of the head when each pulse sends it Where the digital mobile emits electromagnetic radiation whose frequency delights the importance of a device in the form of pulses and enters from the pulse to 546 microseconds and the pulse repetition rate is 215 hertz. Sleeping and tinnitus, resulting in a high rate of cancerous transformation in the body to four percent of the normal rate. The German professor said that cancer in an adult human, resulting from the impact of environmental hazards, can only be discovered after more than ten years have passed since the beginning of the exposure. The report also states that Stuart states that it has not been scientifically proven the occurrence of brain cancer as a result of exposure for electromagnetic waves.

\subsection{Auditory nerve cancer}

A study conducted by the Swedish Karolinska Institute on 750 people revealed that the risk of nerve neoplastic tumors increased by an average of 3.9 times due to use the mobile. In general, more than ten years of people using mobile phones It has increased by 1.9 times. It is mentioned that auditory nerve tumors are a type of benign tumor that may cause brain and nerve damage. The study showed that the risk of injury in people who did not use the mobile for a period of long use it down ear not effecting on nerve the research team discovered that 150 of the people who underwent for the study, the 750 people received tumors of the auditory nerve the use of a mobile phone almost a decade led to the injury of at least one out of every 11 people surveyed. Certainly, the risk increases with the passage of time from the use of mobile phones, that phone users do not feel the mistake of a lock used by earphones, as our research showed that the risk increases from the side on which the mobile phone is placed on-ear.

\subsection{Impotence reproductive organs}

The study was showing by the American Clinic Cleveland that the excessive exposure to electromagnetic waves causes the damage of sperms and an indicated of a few active severities in these people compared to those who are exposed to them in moderation. Those who are exposed to electromagnetic waves in an excessive way, it is due to the effect of radiation, which has a very harmful effect on DNA, which in turn affects the testicular cells that produce the hormone testosterone or the tubes produced by the sperm. The researcher pointed out that the study did not prove the devastating effect of electromagnetic waves on sperm, of testicle tubes, as confirmed by Dr. Clary and his colleague, Bhar in 1998, that waves from the cellphone reduced the numbers of sperms in pottery by a large percentage.

\subsection{Effect EMR on DNA}

Frequent exposure to DNA within the living cell nucleus of DNA to microwave waves, including mobile phone waves, leads to degradation and destruction in the chemical chain of acid, especially brain cells. This came in a research by Dr. Sarkar and his colleagues in 1994 and then by Dr. Daniel and his colleagues in 1994 when he presented the nematode worms to the microwave waves emitted by the Nokia 2115 device and the worm cells showed to break down and DNA damage in inside the nucleus as well as the DNA in the cytoplasm. T.C. and his colleagues also found in 1999 that DNA is under threat and destruction when mobile phone waves of all kinds fall. Lee and Cheng in 1995 and 1996 also concluded that fractions in the DNA chain of any DNA exceeded those in the control sample in the brain cells of mice. 
The tests were done after only two hours since the exposure to the EMR started as for Dr. Phillips in 1999, he linked his dose to exposure to mobile waves and a number of fractures in DNA. There is a direct relationship between them in what is known as the effect relationship with the dose. A group of researchers in Europe found that DNA cells are destroyed if they are exposed to high-frequency radio waves the study indicates that the older cells are more sensitive to lowfrequency waves from electrical lines, as well as the highfrequency waves emanating from the telephone headphones, and a member of the German team, reported that the study showed with the radiation are more effective on age, elderly are more susceptible. These electromagnetic waves are at risk.

\section{TESTING AND RESULT ANALYSIS}

\subsection{RF field strength meter}

RF field strength meter is defined as a device which measures the field strength or power emitted by devices operating in this wave frequency zone and which ensures the intensity of the radio radiation measured in a watt unit divided by a square centimeter $\left(\mu \mathrm{w} / \mathrm{cm}^{2}\right)$. All devices that take readings on any phenomenon or object without direct contact with it are considered devices that are included in remote sensing techniques. So, the electromagnetic radiation intensity meter is intended to measure electric fields and microwaves, Figure 4 , with in a beam starting from ( $30 \mathrm{Mhz}$ to $3 \mathrm{Ghz}$ ). It also has the ability to measure the received energy from radiation sources, which is confined between the values (1.00 to $2000.00) \mu \mathrm{w} / \mathrm{cm}^{2}$. This type of device is considered very sensitive in the readings within the open areas and areas close to other general radiation sources as the device reads the real energy density on the interface of the device.

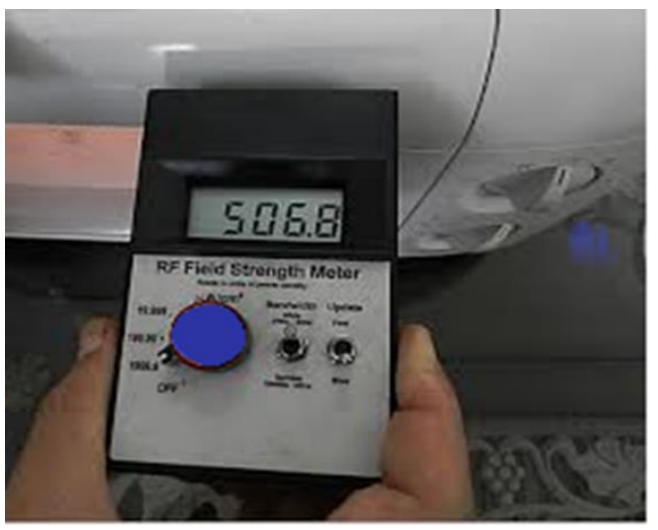

Figure 4. RF field strength meter

Also, this sensitivity gives the same response to the spectrum beam on which this device works and what is meant here is the working frequencies on which the device is designed. To measure, Completely, the device is designed to measure the radio frequencies that come in. It focuses on detecting the electric field, which is very easy to know the polarization in it, which is the vertical extension of the device, that is, the person reading the radiation energy must move the device after several directions to get the best reading or what is meant by the highest intensity of the energy reading. The radiation received from the electric field is due to the fact that most of the radiation sources do not depend in the transmission on a single method for radio transmission systems. There is vertical polarization and horizontal polarization. When moving in the blank, the polarization process changes so it is inclined or circular polarization here.

It should focus on not adopting only one reading Where many possibilities are taken until the required readings are reached by measuring a large number of cellular devices available in the local market and frequently traded by people and knowing the power or radiation energy issued by them. It is clear to us that there are differences in the cellular devices in terms of the radiant power when the radio transmission process is added to cellular devices that Is that which the transmission capacity of the radioactive card is little or relatively low. By taking samples from commonly used mobiles in the local markets, four groups have been selected and each group has 10 types of mobile phones.

The tests are carried out on (4) groups of mobile phones commonly used in Iraq (i.e. Baghdad city) through direct cooperation with mobile marketing centers. The radiation readings issued from each mobile in each group are taken and that was by making a call process in the mobile, after which the electromagnetic radiation intensity measuring device measured. The radiation energy is issued by the mobile device is measured in units of $\mu \mathrm{w} / \mathrm{cm}^{2}$ divided. In order to assess the radiation power of each mobile device, various forms are taken into account to each category in order to know whether or not the cell phone has undergone testing and standardization within the standard specification. In general, the findings showed that the values of the radiation power produced by the samples in the same category are very different.

The first group, i.e. Nokia, is considered one of the most popular mobiles. Table 1 and Figure 5 show that the limits of the radiation power are limited between (145.33-862.87 $\mu \mathrm{w} /$ $\mathrm{cm}^{2}$ ), while the permissible limit should be does not exceed $\left(500 \mu \mathrm{w} / \mathrm{cm}^{2}\right)$. Nokia 7 Plus recorded the highest value of the radiation power while the type of Nokia 7.2 comes in last order. It is clear that in terms of radiation power, three types of this group do not exceed the permissible limit.

The second type which is Huawei mobiles, are tested on a group of mobiles (10 types) and the results are between $\left(114.95-642.72 \mu \mathrm{w} / \mathrm{cm}^{2}\right)$, and two types have exceeded the standard permissible limit as shown in the Figure 6 and Table 2. The category of Y9 has been recorded the best, i.e. lowest, value of the radiation power at $114.95 \mu \mathrm{w} / \mathrm{cm}^{2}$ followed by Y9S and Meta 30 at 235.91 and $337.77 \mu \mathrm{w} / \mathrm{cm}^{2}$, respectively.

Figure 7 and Table 3 display the radiation power of group (3), namely Samsung Galaxy. It seems that the radiation power issued by mobiles in this group ranged between (214.56 $447.89 \mu \mathrm{w} / \mathrm{cm}^{2}$ ), noting that this group in most of its devices did not exceed the permissible standard specification regarding transmissions, which are from mobile phones. This group has a low transmission power and it is better to use it to avoid radiation damage, as its values are shown in Table 3 and Figure 7.

As for the fourth group, which is a group (iPhone), where ten samples are taken and measurements are made on them. The radiation power is limited between (228.43- $442.72 \mu \mathrm{w} /$ $\mathrm{cm}^{2}$ ) and it was found that all the models that are measured work within the permissible standard limit and as shown in the Figure 8 and Table 4 . The 11Pro type of this group is the best compared to others because it recorded the lowest value of the radiative energy at $228.43 \mu \mathrm{w} / \mathrm{cm}^{2}$.

After studying the outcomes of the tables for the four groups of different types of mobile phones, it was found that there is 
a difference between the values of the radiation capacity for each mobile phone. Some of them have a low radiation capacity, and some have a radiation capacity close to the permissible limit, and others exceed this limit. There are also other types with the transmission capacity twice the standard limit. Based on the analysis of the results, it was found that there is a superior in more than one group, such as (iPhone) and (Galaxy) where more than $90 \%$ of their devices operate within the standard specification, which is less than $(0.5) \mathrm{mw} /$ $\mathrm{cm}^{2}$. This matter is very important to avoid the harm caused by the mobile's high capabilities to human health.

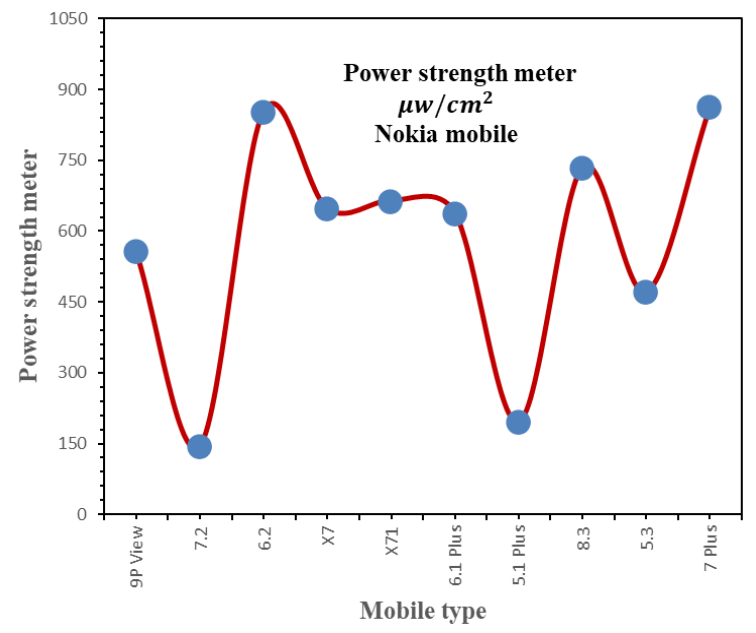

Figure 5. Power strength meter for Nokia mobile group

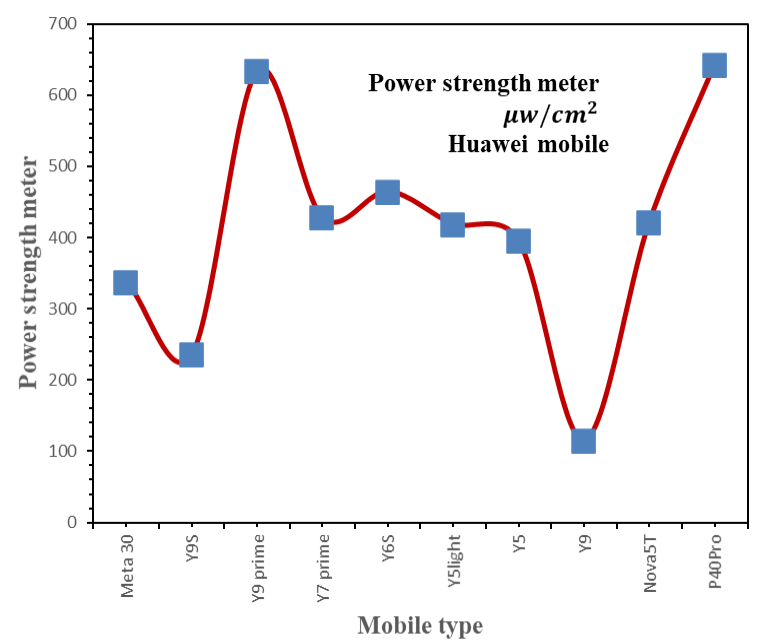

Figure 6. Power strength meter for Huawei mobile group

Table 1. Data for Nokia mobile group

\begin{tabular}{cc}
\hline Power strength meter $\left(\boldsymbol{\mu w} / \mathbf{c m}^{\mathbf{2}}\right)$ & Nokia mobile group \\
\hline 557.72 & $9 \mathrm{P}$ View \\
145.33 & 7.2 \\
853.45 & 6.2 \\
647.74 & $\mathrm{X} 7$ \\
664.23 & $\mathrm{X} 71$ \\
638.65 & 6.1 Plus \\
195.44 & 5.1 Plus \\
734.92 & 8.3 \\
471.31 & 5.3 \\
862.87 & 7 Plus \\
\hline
\end{tabular}

Table 2. Data for Huawei mobile group

\begin{tabular}{cc}
\hline Power strength meter $\left(\boldsymbol{\mu w} \mathbf{c m}^{\mathbf{2}}\right)$ & Huawei mobile group \\
\hline 337.77 & Meta 30 \\
235.91 & Y9S \\
633.37 & Y9 prime \\
427.84 & Y7 prime \\
464.28 & Y6S \\
418.69 & Y5light \\
395.44 & Y5 \\
114.95 & Y9 \\
421.39 & Nova5T \\
642.72 & P40Pro \\
\hline
\end{tabular}

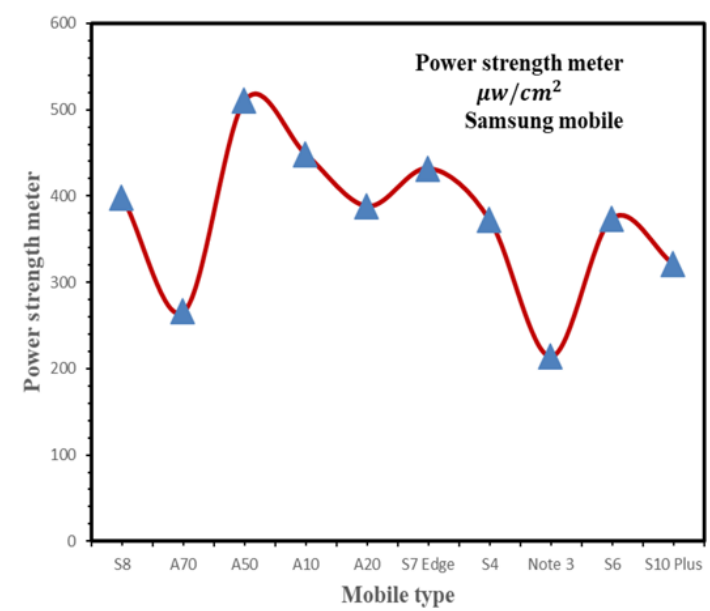

Figure 7. Power strength meter for Samsung mobile group

Table 3. Data for Samsung mobile group

\begin{tabular}{cc}
\hline Power strength meter $\left(\boldsymbol{\mu w} / \mathbf{c m}^{\mathbf{2}}\right)$ & Samsung mobile group \\
\hline 398.24 & S8 \\
266.51 & A70 \\
510.66 & A50 \\
447.89 & A10 \\
388.32 & A20 \\
431.99 & S7 Edge \\
372.12 & S4 \\
214.56 & Note 3 \\
374.22 & S6 \\
321.43 & S10 Plus \\
\hline
\end{tabular}

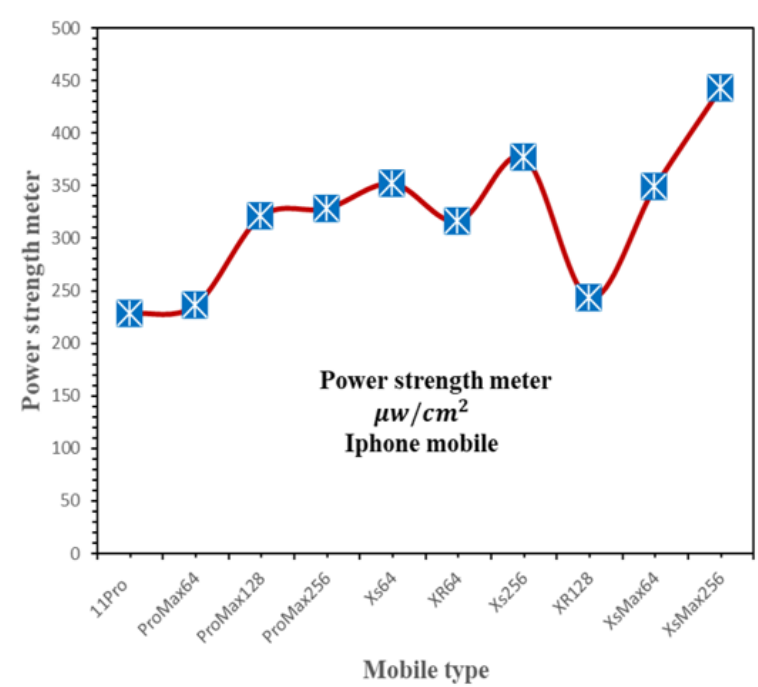

Figure 8. Power strength meter for iPhone mobile group 
Table 4. Data for iPhone mobile group

\begin{tabular}{cc}
\hline Power strength meter $\left(\boldsymbol{\mu w} / \mathbf{c m}^{\mathbf{2}}\right)$ & Nokia mobile group \\
\hline 228.43 & 11 Pro \\
236.97 & ProMax64 \\
321.14 & ProMax128 \\
328.55 & ProMax256 \\
352.17 & Xs64 \\
316.55 & XR64 \\
377.11 & Xs256 \\
243.65 & XR128 \\
349.26 & XsMax64 \\
442.72 & XsMax256 \\
\hline
\end{tabular}

\section{CONCLUSION}

From the results, it becomes clear to us in this aspect that whoever is exposed to radiation less than $0.5 \mathrm{mw} / \mathrm{cm}^{2}$ has a weak effect and this depends on the time of exposure. Also, even if the exposure energy is low, but its time is long, it may become influential. As well, and due to the large number of laboratory experiments, elementary standards have been adopted to determine the value of the radioactive energy its effect on human organs after taking samples from cell phones. It was found that most of these phones give a capacity higher than the acceptable value, and this requires care not to prolong the period of direct exposure to the source of the radiation. As previous experiments showed that exposure to $10 \mathrm{mw} / \mathrm{cm}^{2}$ increases temporarily feeling forgetful and decreased focus with increased stress while exposure to the amount of one 120 $\mathrm{mw} / \mathrm{cm}^{2}$ persons and more directly affects secretions from the pituitary gland and causes the occurrence of impaired fertility in men. But if exposure to $700 \mathrm{mw} / \mathrm{cm}^{2}$ per square centimeter and more, indicating that the output power of the ideal devices is not more than $0.4 \mathrm{mw} / \mathrm{cm}^{2}$ because its effect is very weak for many years.

\section{REFERENCES}

[1] Adeola, A.M., Olwoch, J.M., Botai, J.O., Rautenbach, C.D., Kalumba, A.M., Tsela, P.L., Adisa, O.M., Nsubuga, F.W.N. (2017). Landsat satellite derived environmental metric for mapping mosquitoes breeding habitats in the Nkomazi municipality, Mpumalanga Province, South Africa. South African Geographical Journal, 99(1): 1428. https://doi.org/10.1080/03736245.2015.1117012

[2] Hemba, S., Iortyom, E.T., Ropo, O.I., Daniel, D.P. (2017). Analysis of the physical growth and expansion of Makurdi Town using remote sensing and GIS techniques. Imperial Journal Interdisciplinary Research, 3(7): 821827.

[3] Adefisan, E.A., Bayo, A.S., Ropo, O.I. (2015). Application of geo-spatial technology in identifying areas vulnerable to flooding in Ibadan metropolis.
Journal of Environment and Earth Science, 5(14): 153166.

[4] Ige, S.O., Ajayi, V.O., Adeyeri, O.E., Oyekan, K.S.A. (2017). Assessing remotely sensed temperature humidity index as human comfort indicator relative to land use landcover change in Abuja, Nigeria. Spatial Information Research, 25(4): $523-533$ https://doi.org/10.1007/s41324-017-0118-2

[5] Onamuti, O.Y., Okogbue, E.C., Orimoloye, I.R. (2017). Remote sensing appraisal of Lake Chad shrinkage connotes severe impacts on green economics and socioeconomics of the catchment area. Royal Society Open Science, 4(11): 171120. https://doi.org/10.1098/rsos.171120

[6] Genuis, S.J. (2008). Fielding a current idea: Exploring the public health impact of electromagnetic radiation. Public Health, 122(2): 113-124. https://doi.org/10.1016/j.puhe.2007.04.008

[7] Jorge-Mora, T., Alvarez Folgueiras, M., Leiro-Vidal, J. M., Jorge-Barreiro, F.J., Ares-Pena, F.J., Lopez-Martin, M.E. (2010). Exposure to $2.45 \mathrm{GHz}$ microwave radiation provokes cerebral changes in induction of HSP-90 $\alpha / \beta$ heat shock protein in rat. Progress In Electromagnetics Research, 100: 351-379. https://doi.org/10.2528/PIER09102804

[8] Ghezel-Ahmadi, D., Engel, A., Weidemann, J., Budnik, L. T., Baur, X., Frick, U., Hauser, S., Dahmen, N. (2010). Heavy metal exposure in patients suffering from electromagnetic hypersensitivity. Science of the Total Environment, 408(4): 774-778. https://doi.org/10.1016/j.scitotenv.2009.11.023

[9] Baldi, I., Coureau, G., Jaffré, A., Gruber, A., Ducamp, S., Provost, D., Lebailly, P., Vital, A., Loiseau, H., Salamon, R. (2011). Occupational and residential exposure to electromagnetic fields and risk of brain tumors in adults: A case-control study in Gironde, France. International Journal of Cancer, 129(6): 1477-1484. https://doi.org/10.1002/ijc. 25765

[10] Yakymenko, I., Tsybulin, O., Sidorik, E., Henshel, D., Kyrylenko, O., Kyrylenko, S. (2016). Oxidative mechanisms of biological activity of low-intensity radiofrequency radiation. Electromagnetic Biology and Medicine, 35(2): 186-202. https://doi.org/10.3109/15368378.2015.1043557

[11] Kostoff, R.N., Goumenou, M., Tsatsakis, A. (2018). The role of toxic stimuli combinations in determining safe exposure limits. Toxicology Reports, 5: 1169-1172. https://doi.org/10.1016/j.toxrep.2018.10.010

[12] Melnick, R.L. (2019). Commentary on the utility of the National Toxicology Program study on cell phone radiofrequency radiation data for assessing human health risks despite unfounded criticisms aimed at minimizing the findings of adverse health effects. Environmental Research, 168: 1-6. https://doi.org/10.1016/j.envres.2018.09.010 